\title{
A systematic review and meta-analysis of neostigmine for urinary retention after surgeries
}

\author{
Mengya $\mathrm{Cao}^{1}$, Xiaoxia $\mathrm{Wu}^{2}$, Junmei $\mathrm{Xu}^{1}$ \\ ${ }^{1}$ Department of Anesthesiology, The Second Xiangya Hospital, Central South University, Changsha, China; ${ }^{2}$ Nursing Department, The Third \\ Xiangya Hospital, Central South University, Changsha, China \\ Contributions: (I) Conception and design: M Cao, J Xu; (II) Administrative support: J Xu; (III) Provision of study materials or patients: None; (IV) \\ Collection and assembly of data: M Cao, X Wu; (V) Data analysis and interpretation: M Cao; (VI) Manuscript writing: All authors; (VII) Final \\ approval of manuscript: All authors. \\ Correspondence to: Jummei Xu. Department of Anesthesiology, The Second Xiangya Hospital, Central South University, Changsha 410011, China. \\ Email: 13975148864@139.com.
}

Background: The aim of this research is to analyze the efficacy of neostigmine in the treatment of postoperative urinary retention (POUR).

Methods: In this research, we screened multiple databases including PubMed, EMBASE, Web of Science, and Chinese National Knowledge Infrastructure (CNKI). After a systematic search process, data extraction was conducted. Review Manager 5.2 was adopted for meta-analysis, sensitivity analysis, and bias analysis.

Results: After searching for articles, 20 eligible trials including 1,850 patients after surgery were extracted. Our results suggested that the neostigmine group had a higher effective rate for urinary retention than the Chinese traditional and physical therapy group ( $\mathrm{OR}=7.47$, 95\% CI: 4.10-13.59, overall effect $\mathrm{P}<0.001)$. Further subgroup analysis showed that neostigmine acupoint injection was better than neostigmine intramuscular injection. Time to first voiding in the neostigmine acupoint injection group was shorter than that in the neostigmine intramuscular injection group $(\mathrm{MD}=-81.92,95 \% \mathrm{CI}:-130.13$ to -33.70 , overall $\mathrm{P}<0.001, \mathrm{I}^{2}=99 \%$ with random effects model). Furthermore, neostigmine acupoint injection improved urine excretion ( $\mathrm{MD}=243.40$, 95\% CI: 201.62-285.18, overall $\mathrm{P}<0.0001)$ and reduced the residual urine volume ( $M D=-41.31,95 \% \mathrm{CI}:-58.05$ to -24.58 , overall $\mathrm{P}<0.001, \mathrm{I}^{2}=75 \%$ with random effects model). The results of the sensitivity analysis and publication bias showed that this research was robust and had little publication bias.

Discussion: Our meta-analysis results suggest that neostigmine can effectively improve the symptoms of POUR and neostigmine acupoint injection may achieve a better therapeutic effect.

Keywords: Neostigmine; postoperative urinary retention (POUR); urinary retention; meta-analysis

Submitted Nov 05, 2021. Accepted for publication Jan 20, 2022.

doi: 10.21037/tau-22-16

View this article at: https://dx.doi.org/10.21037/tau-22-16

\section{Introduction}

Urinary retention usually occurs after anesthesia and surgery, with a reported incidence of $5 \%$ to $70 \%$ (1). Risk factors of urinary retention include anesthetics, type of surgery, postoperative local inflammation, age over 50 , and relative immobility after surgery (2). Postoperative urinary retention (POUR) is often considered as a minor postoperative adverse effect and inhibition of micturition reflex after general anesthesia or spinal anesthesia is the main cause of POUR. However, without treatment, POUR can lead to excessive bladder dilatation, acute renal injury, and detrusor injury. These events may result in delayed discharge from hospital and additional care after discharge (3).

Though POUR can be easily managed by catheterization, 
catheterization also increases the risk of urinary tract infection and may lead to further complications such as prosthetic joint infection (4). Other interventions to prevent or treat POUR include medications (e.g., cholinergic drugs and $\alpha$-adrenergic blockers), massage, acupuncture, and hot compress. Interventions targeting anesthesia and analgesia are potential preventive strategies. The prevention of POUR and alternative treatments to catheterization can relieve postoperative morbidity and reduce complications, consequently improving patients' dignity, comfort, and mental health (3).

Neostigmine is a parasympathomimetic drug that acts as a reversible acetylcholinesterase inhibitor. Aeschlimann and Reinert first synthesized it in 1931. Neostigmine indirectly stimulates nicotinic and muscarinic receptors by interfering with the decomposition of acetylcholine (5). Studies have reported that neostigmine is a convenient, safe, and effective drug for treating patients with POUR. When it was injected through the Zusanli acupoint, the effect of neostigmine was better than that of intramuscular injection (6). However, Tomaszewski et al. (7) analyzed the influence of neostigmine hydrochloride administration on the incidence of POUR in orthopedic patients under spinal anesthesia, and no satisfactory results were found. They observed that the incidence of urinary retention in the neostigmine group was higher than that of the control group, presumably resulting from increased bladder smooth muscle tension. These findings show that the use of neostigmine in POUR remains inconclusive. Therefore, in this study, we searched and collected relevant reports to comprehensively analyze the efficacy of POUR. This is a large scale meta-analysis on this topic, and it evaluated details of effects and adverse events to conduct this update research. We present the following article in accordance with the PRISMA reporting checklist (available at https://tau.amegroups.com/article/ view/10.21037/tau-22-16/rc).

\section{Methods}

\section{Literature search strategy}

We searched PubMed, Web of Science, Embase, and the Chinese National Knowledge Infrastructure (CNKI) database for randomized controlled trials (RCTs) published from Jan 1, 2000 to Sep 1, 2021 using the following search terms: (I) neostigmine; (II) urine retention OR POUR; (III) clinical effects. The search strategy involved Medical
Subject Headings (MeSH) and text words combined through Boolean operators "AND".

We conducted a comprehensive search across several databases without restricting for language or publication status. In order to maximize the specificity and sensitivity of the search, the authors also referred to the list of references retrieved, looking for other relevant studies not found through the search strategy.

\section{Study selection}

Potentially relevant articles were reviewed in full to ensure that they satisfied all of the following inclusion criteria: (I) research comparing patients receiving neostigmine or standard therapy; (II) research comparing patients receiving neostigmine acupoint injection or neostigmine intramuscular injection; (III) patients with POUR; (IV) studies containing indicators evaluating effectiveness or other relevant indicators between neostigmine and standard therapy; $(\mathrm{V})$ available in full text.

Studies were excluded based on the following predetermined exclusion criteria: (I) research on other diseases; (II) comparison of other interventions; (III) studies lacking available data; (IV) review, abstract, or duplicate publication.

\section{Data extraction and quality assessment}

Two pairs of reviewers independently screened titles, abstracts, and full-text articles of potentially eligible studies and resolved disagreements through discussion.

The following data parameters were extracted: name of primary author, country(s) of study, patient population under study, number of participants in each arm, patient age [mean and standard deviation (SD)], patient sex, characteristics of the pharmaceutical intervention (dosage and duration of therapy) in each arm, follow-up duration, and outcome measures for each arm.

The validity of eligible RCTs was assessed using the Cochrane risk of bias tool in Review Manager 5.2. Egger's tests and funnel plots were used to evaluate the risk of bias across studies.

\section{Statistical analysis}

Review Manager (version 5.2, Cochrane Collaboration, 2011) was used to estimate the impact of the results in the selected reports. 


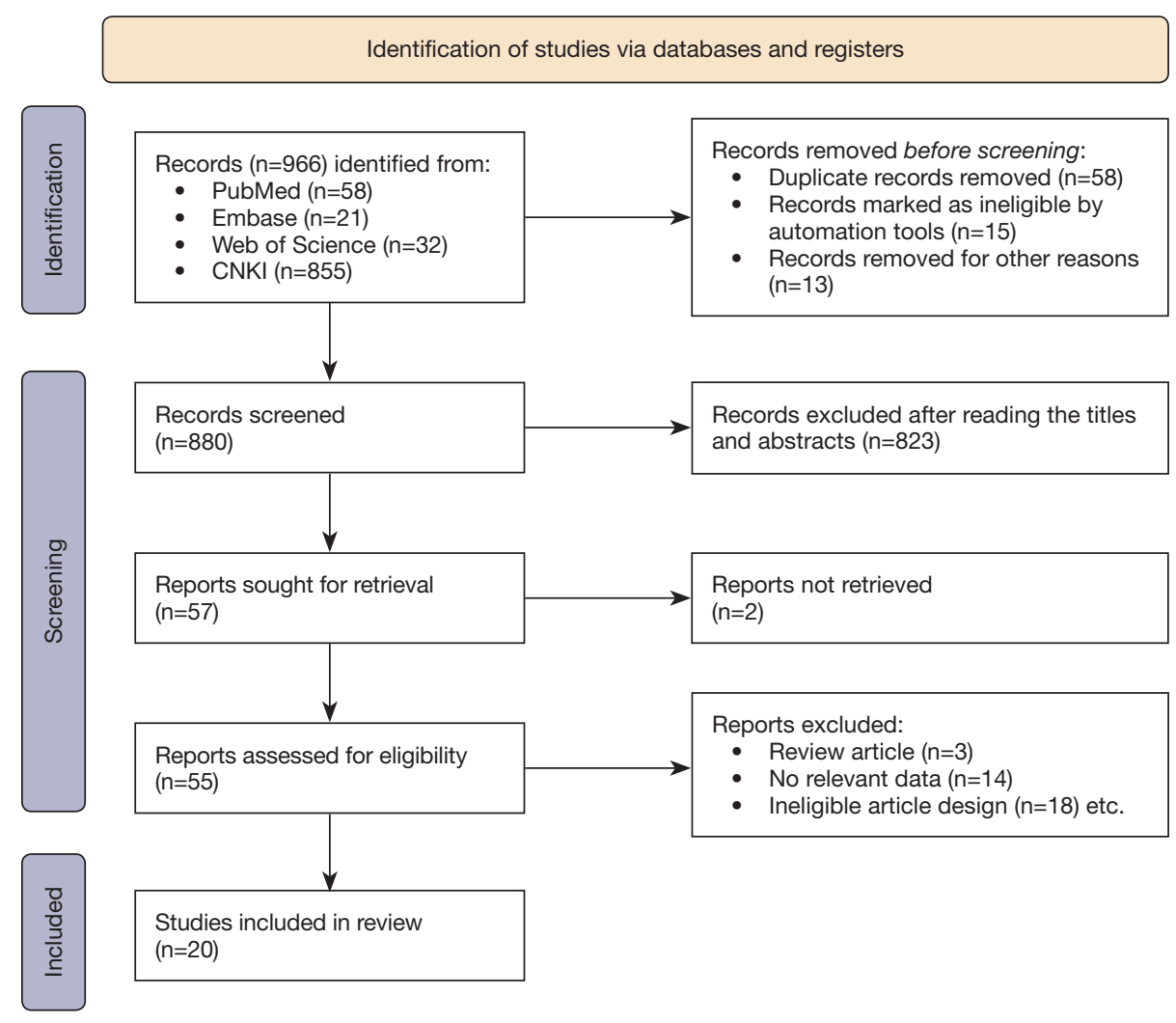

Figure 1 PRISMA flowchart detailing the search strategy for study inclusion.

To measure the consistency of the effect size [odds ratio $(\mathrm{OR})$ and mean difference (MD)], pairwise meta-analyses were performed with a DerSimonian and Laird random effects model to calculate the pooled estimates of OR and MD with $95 \%$ CIs of direct comparisons between the experimental group and control group.

Heterogeneity of $0 \%$ to $40 \%$ was considered as "might not be important," $30 \%$ to $60 \%$ as "moderate heterogeneity," $50 \%$ to $90 \%$ as "substantial heterogeneity," and $75 \%$ to $100 \%$ as "considerable heterogeneity." If $\mathrm{P}<0.05$ or $\mathrm{I}^{2}>50 \%$, the random effects model would be used for analysis. If $\mathrm{P} \geq 0.05$ and $\mathrm{I}^{2} \leq 50 \%$, the fixed effects model would be used for analysis. When heterogeneity was present, the random effects model was used to calculate the pooled OR, whereas the fixed effects model was used in its absence.

Publication bias was examined by visual inspection of funnel plots and by using Egger's tests. Sensitivity analysis was conducted by omitting a single study each time to observe the influence of the individual outcome on the overall analysis.

\section{Results}

\section{Search process}

The initial search yielded 966 articles from 4 databases including PubMed, Embase, Web of Science, and CNKI. After the first screening, 880 records remained. By screening the titles and abstracts, an additional 823 records were excluded because they were review articles, letters, case reports, comments, or editorials. Subsequently, 57 articles remained.

Of these, 37 articles were further excluded due to various reasons including different study designs or insufficient data available. Ultimately, 20 studies met the inclusion criteria and were included in the present meta-analysis, with a total of 1,850 patients. This process, which followed the PRISMA guidelines (8), including the reasons for excluding studies, is illustrated in Figure 1.

\section{Characteristics of the included studies}

Table 1 lists the main characteristics of the 20 included trials 


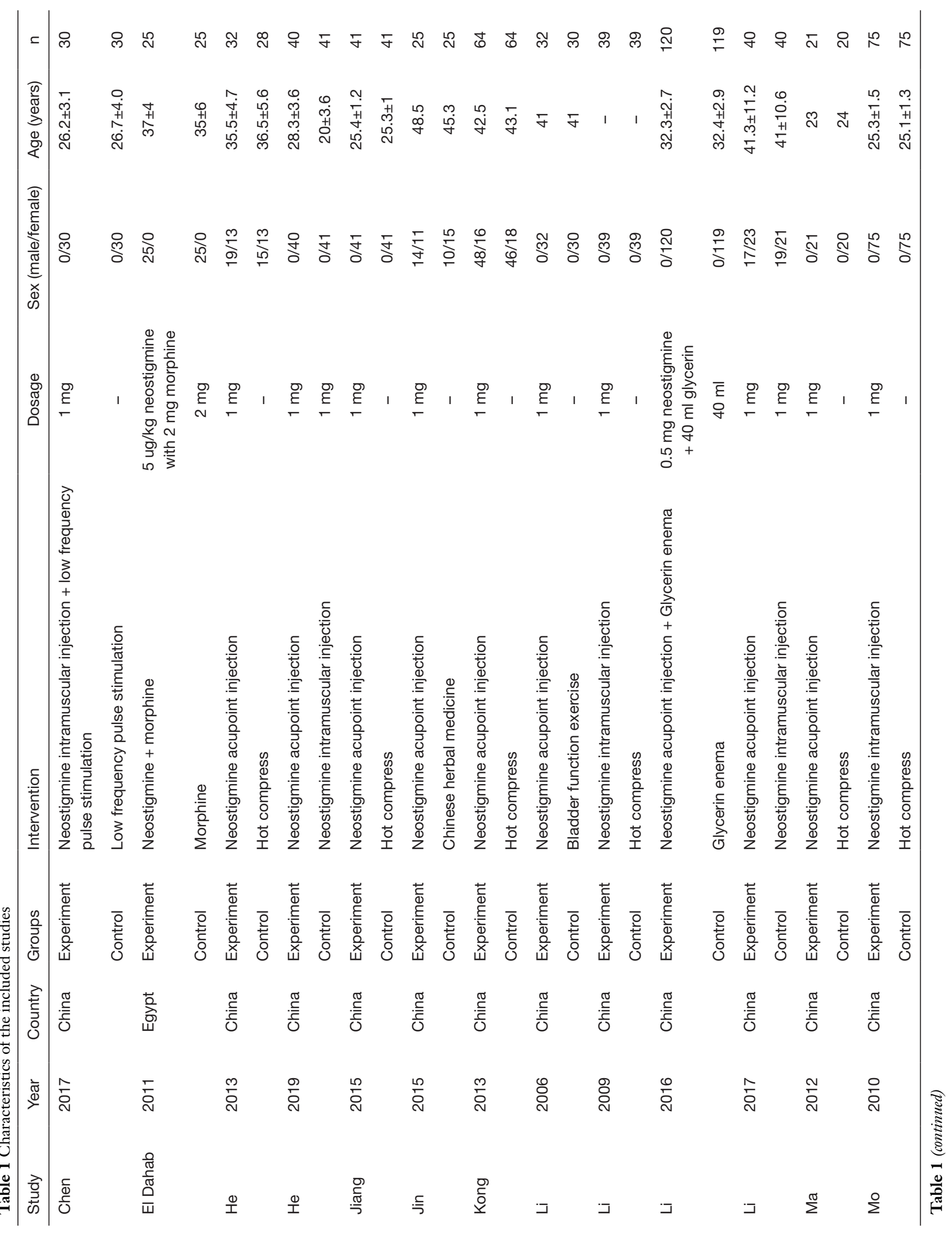




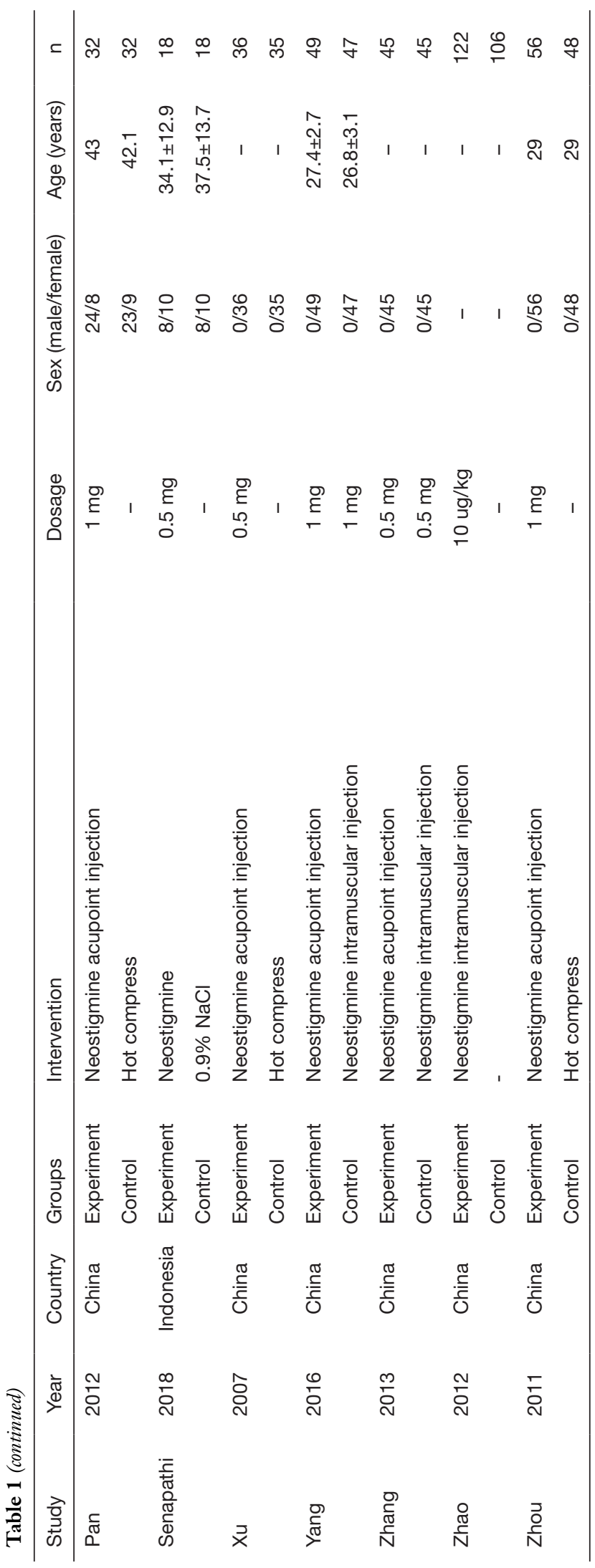

(9-28). All 20 articles were published from 2006 to 2019. The experimental interventions included neostigmine injection (6 trials, 30\%), neostigmine injection with physiotherapy (12 trials, $60 \%$ ), and neostigmine injection combined with other drug treatments ( 2 trials, $10 \%$ ). Correspondingly, the control groups received usual Chinese traditional therapy (hot compress, low frequency pulse stimulation, and Chinese traditional medicine) and physical therapy (functional exercise), or drugs such as morphine. These studies contained a total of 1,850 patients $(942$ patients in the experimental groups and 908 patients in control groups). The sample size was between 36 and 239 .

\section{Results of quality assessment}

The Cochrane risk of bias assessment tool was used to evaluate the risk of bias of the included studies. Among the 20 articles, high risk of selection bias, performance bias, detection bias, attrition bias, reporting bias, and other bias were found in 8 different studies (Figure 2).

In view of the bias summary, only 1 to 2 trials showed bias (Figure 3). Visual inspection of the funnel plot of studies reporting the effectiveness rate showed some asymmetry, and Egger's test indicated that there was little evidence of publication bias.

\section{Results of the heterogeneity tests}

Heterogeneity analysis of the effective rate for urinary retention between the experimental and control groups A meta-analysis of the difference in effective rate was conducted. The overall result showed that the experimental group had a higher effective rate than the control group (OR $=7.47$, 95\% CI: 4.10-13.59, overall effect $\mathrm{P}<0.00001$, $\mathrm{I}^{2}=73 \%$ random effects model) (Figure 4 ). Based on the injection methods of neostigmine, subgroup differences including neostigmine $v s$. control and neostigmine acupoint injection $v s$. neostigmine intramuscular injection were analyzed. The subgroup analysis results showed that neostigmine was significantly better than usual Chinese traditional and physical therapies for urinary retention $(\mathrm{OR}=8.56,95 \%$ CI: 4.12-17.81, overall effect $\mathrm{P}<0.00001$, $\mathrm{I}^{2}=77 \%$ random effects model). In addition, neostigmine acupoint injection had a higher effective rate than neostigmine intramuscular injection for urinary retention $(\mathrm{OR}=4.42$, 95\% CI: $1.78-10.96$, overall effect $\mathrm{P}=0.001$, $\mathrm{I}^{2}=41 \%$ fixed effects model). 


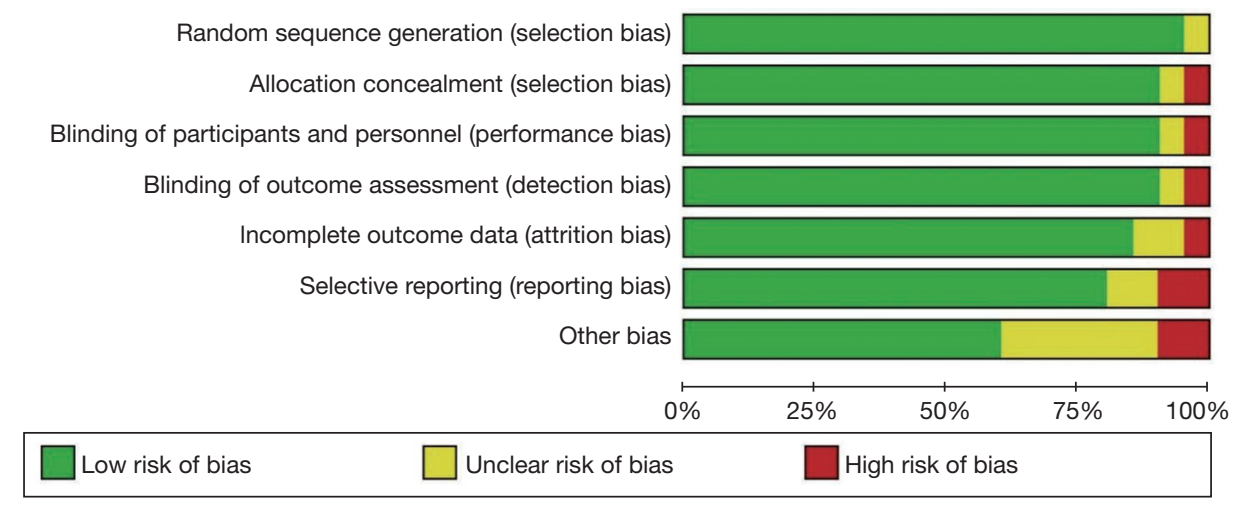

Figure 2 Graph of the risk of bias.

\section{Heterogeneity analysis of the time to first voiding between the experimental and control groups}

Similarly, a meta-analysis for the time to first voiding (min) between the experimental and control groups was performed. The overall result showed that the time to first voiding in the experimental group was shorter than that of the control group ( $M D=-81.92,95 \% \mathrm{CI}:-130.13$ to -33.70 , overall $\mathrm{P}=0.0009, \mathrm{I}^{2}=99 \%$ with random effects model) (Figure 5). The subgroup analysis showed that neostigmine had a better effect for urinary retention (MD $=-47.50,95 \%$ CI: -60.83 to -34.17 , overall $\mathrm{P}<0.00001$ ), while acupoint injection of neostigmine had a better effect than intramuscular injection $(\mathrm{MD}=-95.92,95 \% \mathrm{CI}$ : -161.74 to -30.10 , overall $\mathrm{P}=0.004, \mathrm{I}^{2}=99 \%$ with random effects model).

\section{Heterogeneity analysis of residual urine volume between the experimental and control groups} Four studies covered residual urine volume. The overall results showed that neostigmine treatment could reduce residual urine volume $(\mathrm{MD}=-55.43,95 \% \mathrm{CI}:-80.90$ to $-29.96, \mathrm{P}<0.00001, \mathrm{I}^{2}=89 \%$ with random effects model) (Figure 6). The subgroup analysis showed that neostigmine acupoint injection resulted in significantly less residual urine volume than the neostigmine intramuscular injection group $(\mathrm{MD}=-41.31,95 \% \mathrm{CI}:-58.05$ to -24.58 , overall $\mathrm{P}<0.00001$, $\mathrm{I}^{2}=75 \%$ with random effects model). However, neostigmine resulted in less residual urine volume than usual care (MD $=-78.84,95 \%$ CI: -101.46 to $-56.23, \mathrm{P}<0.00001, \mathrm{I}^{2}=2 \%$ with random effects model), and this result might be attributed to the insufficient article sample size.
Heterogeneity analysis of the volume of urine excreted between the experimental and control groups

To better evaluate the efficacy of neostigmine, we collected data on the volume of urine excreted $(\mathrm{mL})$. Only 1 article reported the volume of urine excreted in each subgroup. The overall results showed that the experimental group had less residual urine volume than the control group (MD $=98.71,95 \%$ CI: -185.49 to $382.90, \mathrm{P}<0.00001, \mathrm{I}^{2}=99 \%$ with random effects model) (Figure 7). In the subgroup analysis, neostigmine and the control group had no difference in the volume of urine excreted ( $M D=-46.60$, 95\% CI: -95.87 to $2.67, \mathrm{P}=0.06$ ). Neostigmine acupoint injection resulted in a greater volume of urine excreted than the neostigmine intramuscular injection group (MD $=243.40,95 \%$ CI: 201.62 to 285.18 , overall $\mathrm{P}<0.00001$ ).

\section{Results of sensitivity analysis and publication bias}

To evaluate the sensitivity of the included articles, we omitted a single study each time to observe the influence of the individual outcome on the overall effectiveness of urinary retention. In Figure 4, the results showed high heterogeneity, with $\mathrm{I}^{2}=73 \%$. When Mo et al.'s article in 2012 was removed, $\mathrm{I}^{2}$ had the biggest change to $75 \%$, which indicated the robustness of the included research (Figure 8).

We generated a funnel plot to evaluate the effectiveness rate of urinary retention, and visually the results showed that the shape was symmetrical. The P-value of Egger's test was 0.245 , which indicated no publication bias existed in this research (Figure 9). 


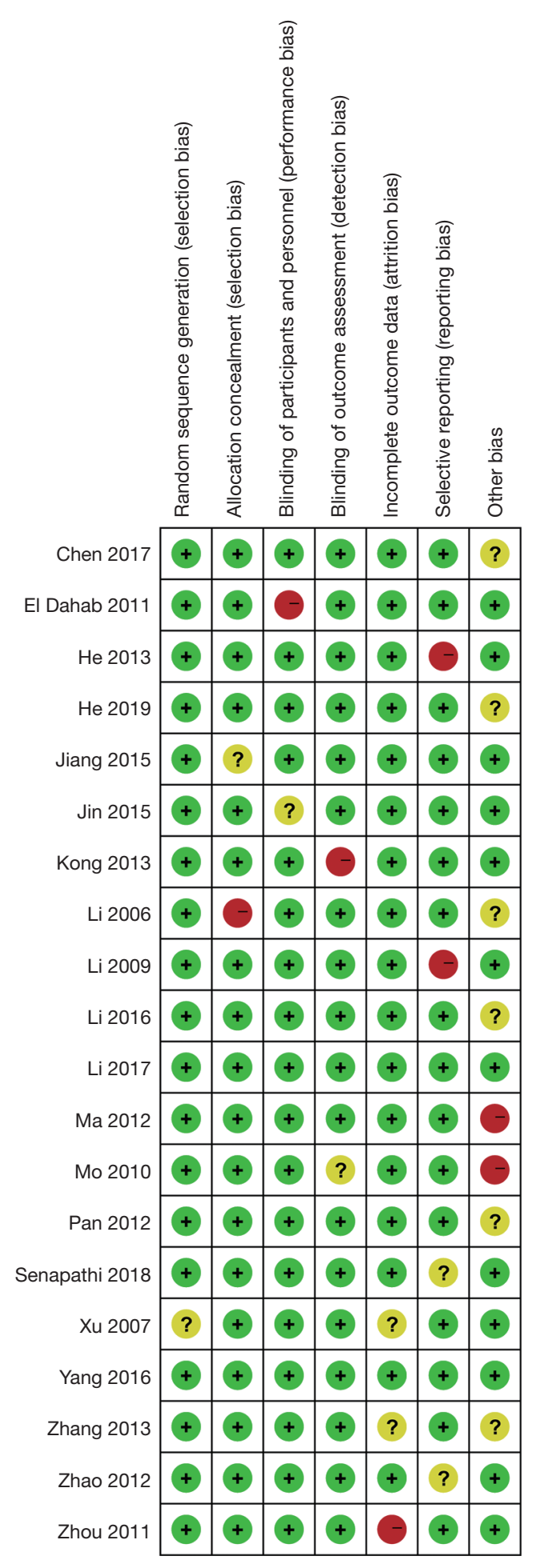

Figure 3 Risk of bias for each study, using 3 colors: green = low risk; yellow with question mark = unclear; and red = high risk.

\section{Discussion}

Our meta-analysis of 20 trials with 1,850 participants evaluated the efficacy of neostigmine in the treatment of urinary retention after surgeries. The indicators included the effective rate, the time to first voiding, residual urine volume, and volume of urine excreted. Our results showed that neostigmine was an effective therapy for POUR. In addition, neostigmine acupoint injection is more effective in treating POUR than neostigmine intramuscular injection.

The parasympathetic nervous system has been reported to play an important role in regulating bladder function. This pathway remains inactive during the filling period and is responsible for micturition through detrusor contraction and sphincter relaxation. Neostigmine is an acetylcholinesterase inhibitor that can inhibit cholinesterase activity and enhance the acetylcholine effect. Neostigmine directly excites the bladder detrusor and causes concentration-dependent contractions, consequently promoting urination. Previous reports showed that neostigmine effectively promoted bladder emptying (7). The researchers found evidence of detrusor overactivity (DO) $(\mathrm{P}=0.031)$ and decreased maximum cystometric capacity (MCC) $(\mathrm{P}=0.056)$ after neostigmine treatment (29). Consistent with our results, the time to first voiding after neostigmine injection was shorter than physical and Chinese traditional therapy, and residual urine volume and volume of urine excreted were significantly lower after neostigmine injection.

Acupuncture is widely accepted in China as an effective therapy for POUR. In traditional Chinese medicine, Zusanli is traditionally considered to be an effective acupoint in treating gastrointestinal and urinary system diseases. Zusanli point is located under the four fingers of the external knee and the edge of the tibia. Agents injected though this acupoint can enhance acupoint stimulation (18), and even normal saline acupoint injection can improve acupoint stimulation and is not considered as normal placebo. Neostigmine can be injected into Zusanli on both sides to coordinate the contraction of bladder smooth muscle (30). Previous research indicated that neostigmine acupoint injection could decrease the onset time and enhance the therapeutic effect (13). Our meta-analysis showed that neostigmine acupoint injection resulted in a greater volume of urine excreted than the neostigmine intramuscular injection group. These findings 


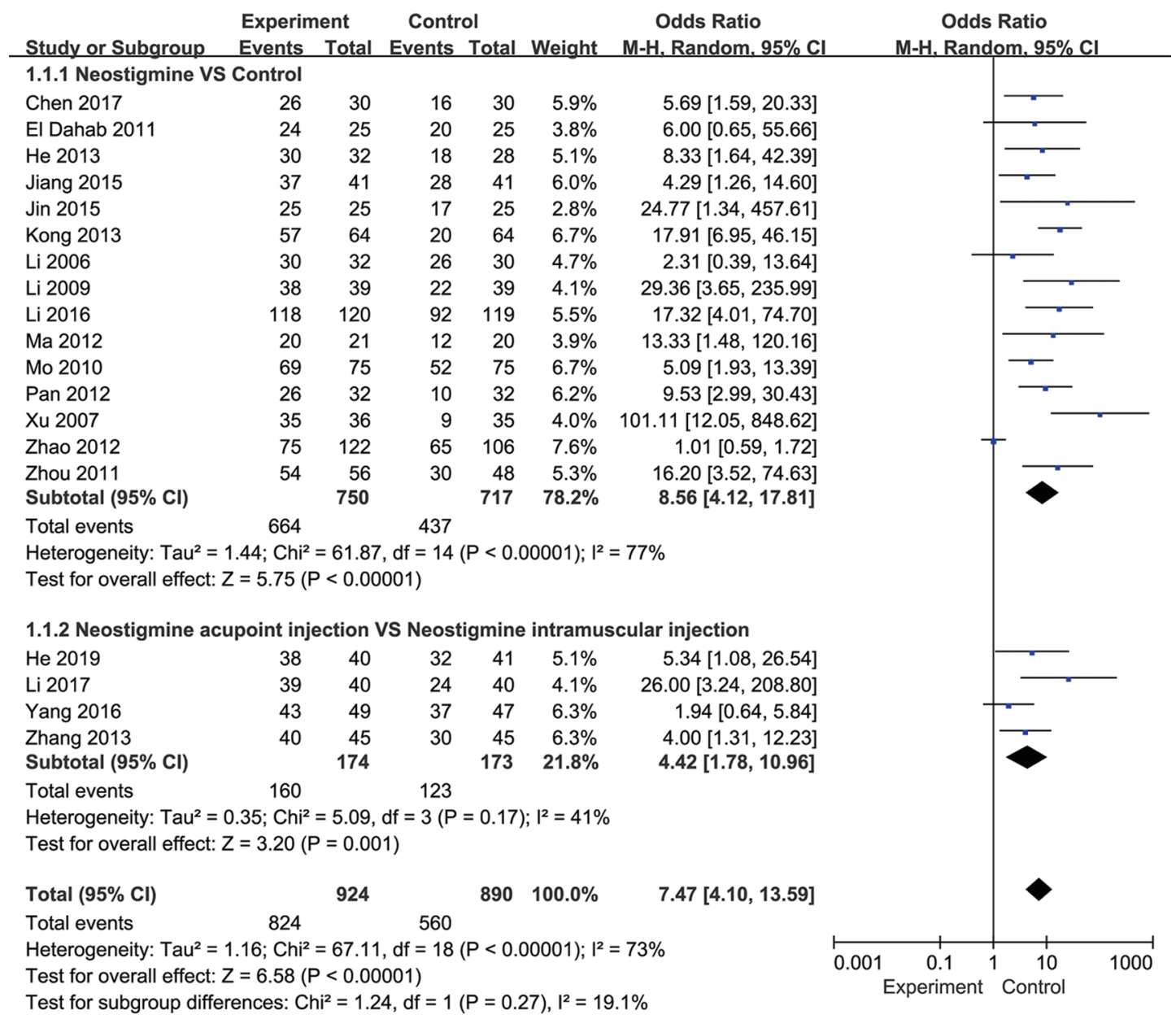

Figure 4 Forest plots for the effective rate of urinary retention in the experimental versus control groups.

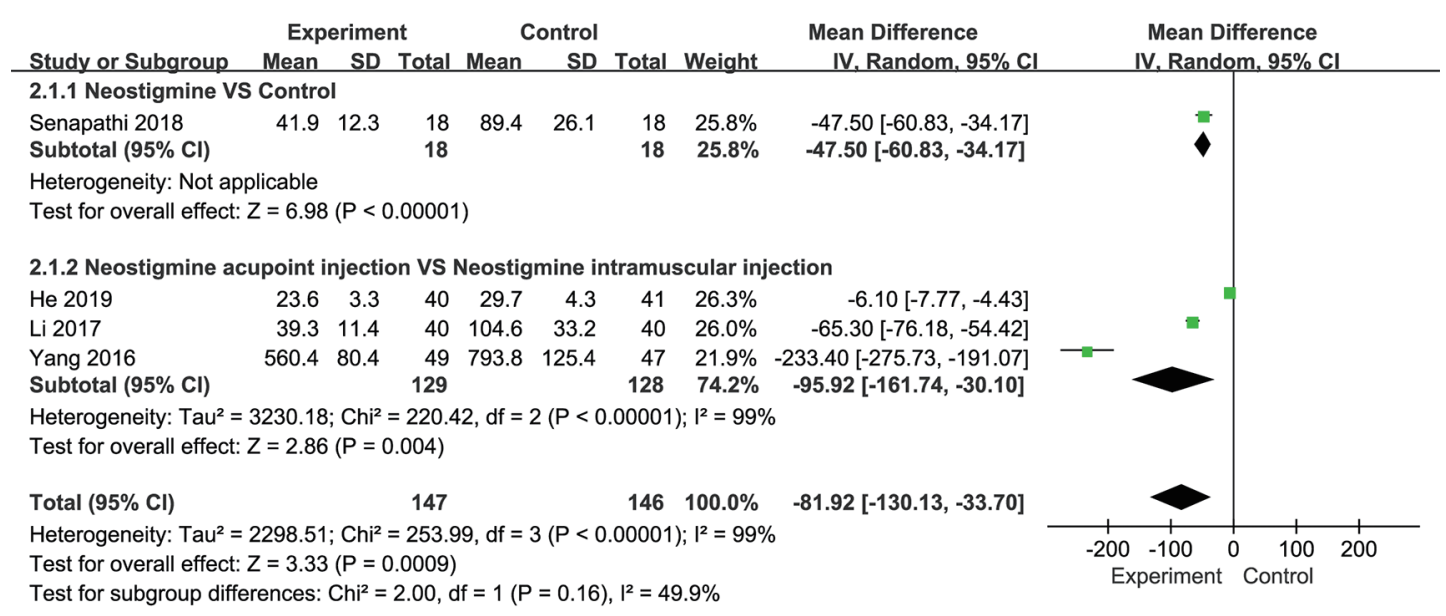

Figure 5 Forest plots for the effects of the time in first voiding in the experimental versus control groups. 


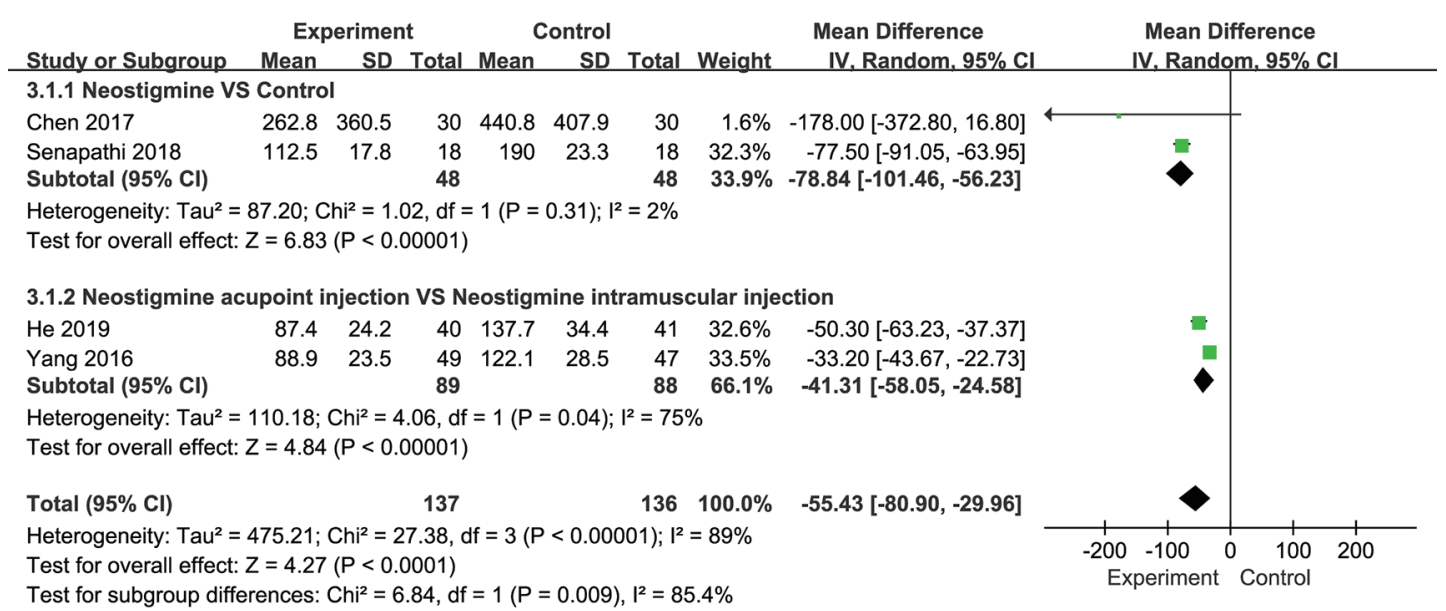

Figure 6 Forest plots for the effects of residual urine volume in the experimental versus control groups.

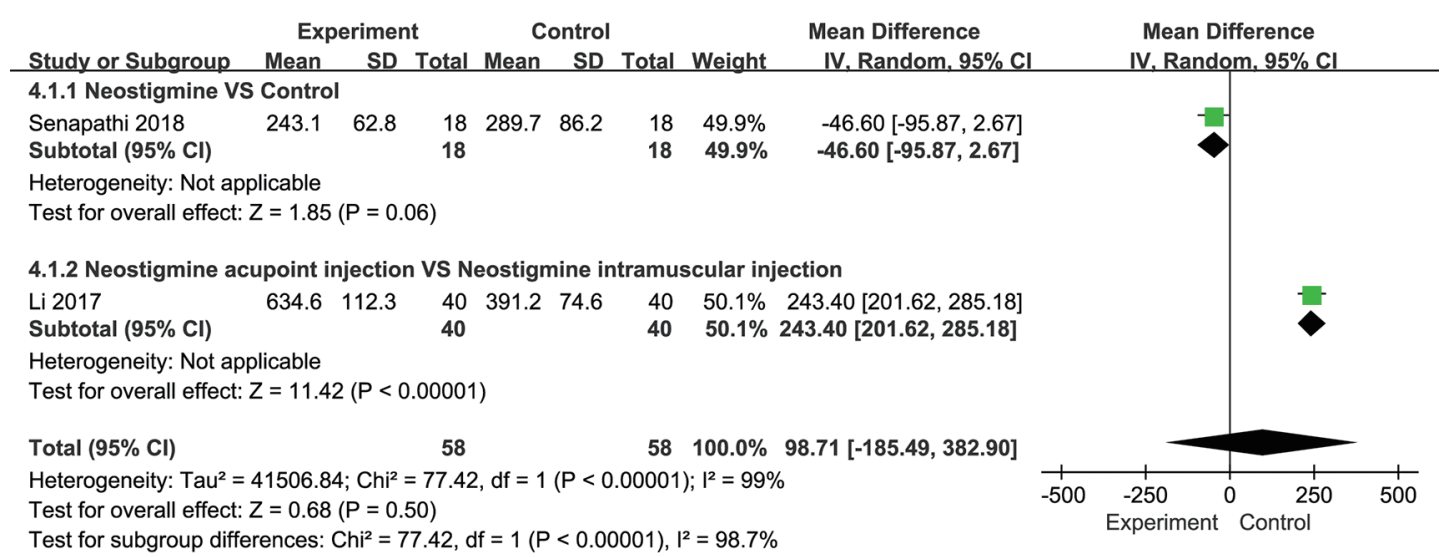

Figure 7 Forest plots for the effects of the volume of urine excreted in the experimental versus control groups.

may help clinicians use transurethral surgery-related pain relief without fear of increased voiding difficulty or acute urinary retention $(31,32)$. Above all, neostigmine is an easily available, proven effective, safe and cheap drug that can be used for POUR.

However, there are still some limitations in this study. Firstly, the neostigmine group and the control group had no difference in volume of urine excreted (Figure 6). This insignificant result might be attributed to the insufficient article sample size. The problem lies in the limitation of available reports. Further research is needed to expand the sample size and draw a scientific conclusion. Secondly, more indicators, especially adverse events or complications, should be included in future analyses. Up to now, there have been limited countries that have conducted research on this topic, and the number of related articles is limited. Therefore, more research from various countries should be included in future research. 


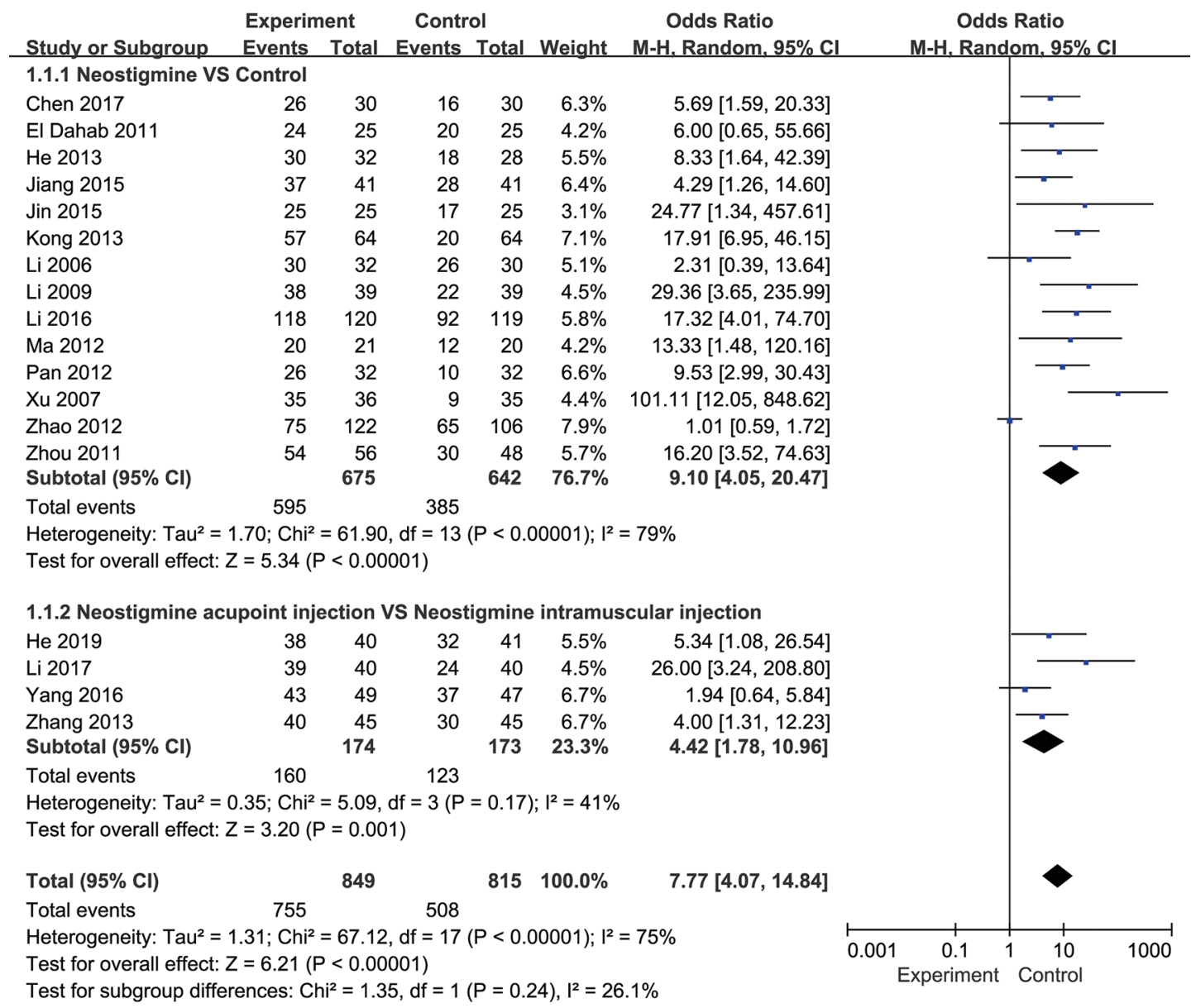

Figure 8 Sensitivity analysis for the effects of urinary retention between the experimental and control groups.

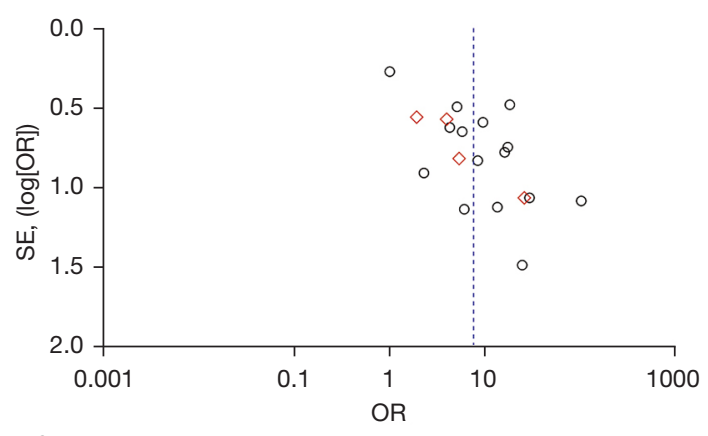

Subgroups
ONeostigmine vs.control
$\diamond$ Neostigmine acupoint injection vs.neostigmine intramuscular injection

Figure 9 Funnel plot of publication bias.

\section{Acknowledgments}

Funding: None.

\section{Footnote}

Reporting Checklist: The authors have completed the PRISMA reporting checklist. Available at https://tau. amegroups.com/article/view/10.21037/tau-22-16/rc

Conflicts of Interest: All authors have completed the ICMJE uniform disclosure form (available at https://tau. amegroups.com/article/view/10.21037/tau-22-16/coif). 
The authors have no conflicts of interest to declare.

Ethical Statement: The authors are accountable for all aspects of the work in ensuring that questions related to the accuracy or integrity of any part of the work are appropriately investigated and resolved.

Open Access Statement: This is an Open Access article distributed in accordance with the Creative Commons Attribution-NonCommercial-NoDerivs 4.0 International License (CC BY-NC-ND 4.0), which permits the noncommercial replication and distribution of the article with the strict proviso that no changes or edits are made and the original work is properly cited (including links to both the formal publication through the relevant DOI and the license). See: https://creativecommons.org/licenses/by-nc-nd/4.0/.

\section{References}

1. 1. Baldini G, Bagry H, Aprikian A, et al. Postoperative urinary retention: anesthetic and perioperative considerations. Anesthesiology 2009;110:1139-57.

2. Chen S, Sun H, Xu H, et al. Effects of Acupuncture on Hospitalized Patients with Urinary Retention. Evid Based Complement Alternat Med 2020;2020:2520483.

3. Jackson J, Davies P, Leggett N, et al. Systematic review of interventions for the prevention and treatment of postoperative urinary retention. BJS Open 2019;3:11-23.

4. Alamanda VK, Springer BD. Perioperative and Modifiable Risk Factors for Periprosthetic Joint Infections (PJI) and Recommended Guidelines. Curr Rev Musculoskelet Med 2018;11:325-31.

5. Kasi PM. The use of intravenous neostigmine in palliation of severe ileus. Case Rep Gastrointest Med 2013;2013:796739.

6. Li HY. Observation of the effect of bilateral Zusanli neostigmine injection on postoperative urinary retention after mixed hemorrhoids surgery. China Diagnosis and Treatment of Anorectal Diseases, 2014.

7. Tomaszewski D, Bałkota M. Intramuscular Administration of Drotaverine Hydrochloride Decreases Both Incidence of Urinary Retention and Time to Micturition in Orthopedic Patients under Spinal Anesthesia: A Single Blinded Randomized Study. Biomed Res Int 2015;2015:926953.

8. Page MJ, McKenzie JE, Bossuyt PM, et al. The PRISMA 2020 statement: an updated guideline for reporting systematic reviews. Syst Rev 2021;10:89.

9. Kong XY. Clinical study on "moxibustion box" moxibustion combined with point injection of neostigmine in treating postoperative urinary retention after anorectal surgery. Acta Chinese Medicine 2013;28:1089-90.

10. El Dahab HA, Samir R, Menesy T, et al. Adding neostigmine to morphine epidurally lessens the incidence of postoperative urine retention: A comparative study. Egyptian Journal of Anaesthesia 2011;27:89-94.

11. Senapathi TGA, Wiryana M, Subagiartha IM, et al. Effectiveness of intramuscular neostigmine to accelerate bladder emptying after spinal anesthesia. Ther Clin Risk Manag 2018;14:1685-9.

12. Zhao D, Huang H, Xiao S, et al. Postoperative selfcontrolled analgesia with caudal and preventing urinary retention by using Neostigmine used in operation of anal disease. Chinese Medical Journal of Metallurgical Industry 2012;29:4-6.

13. Li T. Observation of the effect of Enemia Glycerini combined with Zusanli injection on neostigmine in the treatment of postpartum urinary retention. Journal of Clinical Medical 2016;3:1199-200.

14. He ZH. Treatment of urinary retention after anorectal surgery by injection of Zusanli point with neostigmine methylsulfate. Practical Chinese and Western Medicine 2013;13:33-4.

15. Li ZX, Wang ZG, Yin ZT. Observation on the effect of small Bupleurum Decoction Combined with Zusanli acupuncture combined with neostigmine injection on postoperative urinary retention after anorectal surgery. Modern Journal of Integrated Traditional Chinese and Western Medicine 2017;26:60-2.

16. Mo LX. Clinical study of neostigmine and Enema Glycerini in the treatment of postpartum urinary retention. Chinese Journal of Misdiagnostics 2010;10:71-2.

17. Li X. Effect of neostigmine intramuscular injection plus bladder massage on postpartum urinary retention. Nursing Practice and Research 2009;6:26-7.

18. Chen HZ, Tang QL, Li X, et al. Application of neostigmine combined with low frequency pulse stimulation in urinary retention after spontaneous labor. Chinese Nursing Research 2017;31:2771-2.

19. Li N, Li Y, Yang JB. Neostigmine acupoint blocking for 32 cases of postoperative urinary retention after cervical cancer surgery. Journal of Shaanxi University of Chinese Medicine 2006;(05):44-5.

20. Yang GQ, Li TJ. Observation of the effect of neostigmine acupoint injection on postpartum urinary retention. Journal of Clinical Acupuncture and Moxibustion 
2016;32:35-7.

21. Jiang H. Observation on the effect of neostigmine acupoint injection in the treatment of postpartum urinary retention. Shenzhen Journal of Integrated Traditional Chinese and Western Medicine 2015;25:137-8.

22. Ma L. Clinical observation of neostigmine injection in the treatment of postpartum urinary retention. Chinese Community Doctors 2012;14:146.

23. Jin XD. Clinical observation of neostigmine acupoint injection in treating postoperative urinary retention after anorectal diseases. New Chinese Medicine 2015;47:183-4.

24. He L, Long R, Liu YH, et al. Nursing effect of neostigmine injection on postpartum urinary retention. Electronic Journal of Practical Clinical Nursing 2019;4:44-5.

25. Pan LJ. Acupuncture combined with acupoint injection of neostigmine for 32 cases of postoperative urinary retention after hemorrhoids surgery. Journal of Emergency in Traditional Chinese Medicine 2012;21:790.

26. Xu CF. Injection of neostigmine injection at Zusanli point for 36 cases of postpartum urinary retention. Modern Journal of Integrated Traditional Chinese and Western Medicine 2007:2253-4.

27. Zhou LY, Yang HJ, Pang JL. Clinical observation of

Cite this article as: $\mathrm{Cao} M, \mathrm{Wu} \mathrm{X}, \mathrm{Xu} \mathrm{J}$. A systematic review and meta-analysis of neostigmine for urinary retention after surgeries. Transl Androl Urol 2022;11(2):190-201. doi: 10.21037/ tau-22-16 injection of neostigmine injection at Zusanli point for postpartum urinary retention. Contemporary Medical Symposium 2011;9:156-7.

28. Zhang ZY. Observation of the effect of neostigmine injection at Zusanli on postpartum urinary retention. Clinical Journal of traditional Chinese Medicine 2013;25:503.

29. Lei C. Clinical observation and nursing experience of auricular point sticking combined with Zusanli point injection neostigmine in the treatment of postpartum urinary retention. Journal of Guangzhou University of traditional Chinese Medicine 2014;31:59-61.

30. Agarwal MM, Singh SK, Batra YK, et al. Effect of lumbar epidural administration of neostigmine on lower urinary tract function. Neurourol Urodyn 2010;29:443-8.

31. Lau H, Brooks DC. Predictive factors for unanticipated admissions after ambulatory laparoscopic cholecystectomy. Arch Surg 2001;136:1150-3.

32. Scott AJ, Mason SE, Langdon AJ, et al. Prospective Risk Factor Analysis for the Development of Post-operative Urinary Retention Following Ambulatory General Surgery. World J Surg 2018;42:3874-9.

(English Language Editor: C. Betlzar) 